Supporting Information to

\title{
Operando Analysis of a Lithium/Sulfur Battery by Small Angle Neutron Scattering
}

Sebastian Risse ${ }^{{ }^{\dagger}}$, Eneli. Härk ${ }^{a}$, Ben Kent ${ }^{a}$ and Matthias Ballauff ${ }^{a, b}$

${ }^{a}$ Institute for Soft Matter and Functional Materials, Helmholtz-Zentrum Berlin für Materialien und Energie, Hahn Meitner Platz 1, 14109 Berlin, Germany.

b Institute of Physics, Humboldt-University Berlin, Unter den Linden 6, 10099 Berlin, Germany.

${ }^{+}$Corresponding author: sebastian.risse@helmholtz-berlin.de. 
The limits of the structure size that can be analyzed with the chosen q-range of $0.63-8 \mathrm{~nm}^{-1}$ were estimated by using the scattering form factor, $\boldsymbol{P}(\boldsymbol{q})$, of poly dispersed spheres (pds) and assuming a structure factor, $\boldsymbol{S}(\boldsymbol{q})$, of one which is valid for diluted systems. The resulting scattering intensities with an added incoherent background are shown in figure S1a.

a)



b)

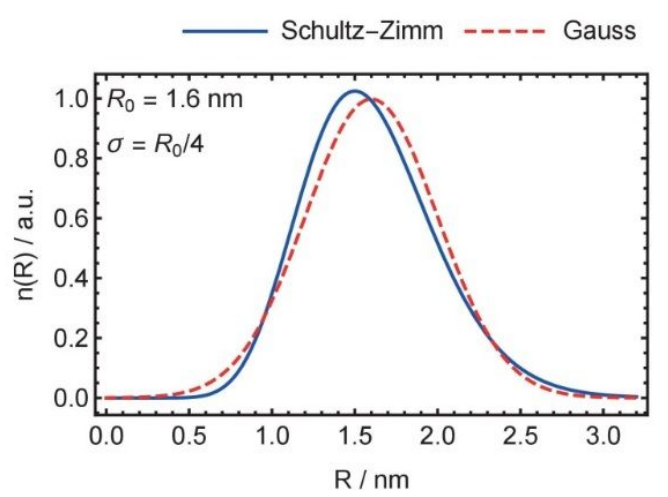

Fig. S1 a) Simulated SANS curves for polydispersed spheres with different diameters by using a Schultz-Zimm distribution with a value for the polydispersity $\sigma$ of $25 \%$. An incoherent background was also added. The dashed lines indicate the q-range of the operando experiment. b) Schultz-Zimm distribution versus Gauss distribution with $25 \%$ polydispersity and an average particle radius of $1.6 \mathrm{~nm}$.

$$
\frac{\mathrm{d} \Sigma_{\text {pds }}}{\mathrm{d} \Omega}(q)=P(q) S(q) \text { with } S(q) \approx 1
$$

The polydispersity was introduced by making use of the Schultz-Zimm distribution, $n(R)$, which yields analytical expressions. The asymmetry of this distribution is the lower the smaller the polydispersity parameter $\sigma$ is.

$$
P(q)=\int_{0}^{\infty} n(R)\left|\Delta S L D V_{\mathrm{p}} \frac{3(\sin (q R)-q R \cos (q R))}{(q R)^{3}}\right|^{2} \mathrm{~d} R
$$

Here $V_{\mathrm{p}}$ is the volume of a single spheres and $R$ the radius of the sphere. The Schultz-Zimm equation is defined as follows:

$$
n(R)=\left(\frac{z+1}{R_{0}}\right)^{z+1} \frac{R^{z}}{\Gamma(z+1)} \exp \left(-\frac{z+1}{R 0} R\right)
$$

Here $Z=\left(\frac{R_{0}}{\sigma}\right)^{2}-1, \sigma=\frac{R_{0}}{4}, R_{0}$ the average radius and $\Gamma(z+1)$ the gamma function. Figure $\mathbf{S} \mathbf{1} \mathbf{b}$ shows the Schultz-Zimm distribution and the Gaussian distribution for the same polydispersity of $25 \%$ and an average particle radius of $1.6 \mathrm{~nm}$.

Figure S2 shows scanning electron microscopy images of the cloth-like material ACN-157-15 by Kynol ${ }^{\circledR}$.

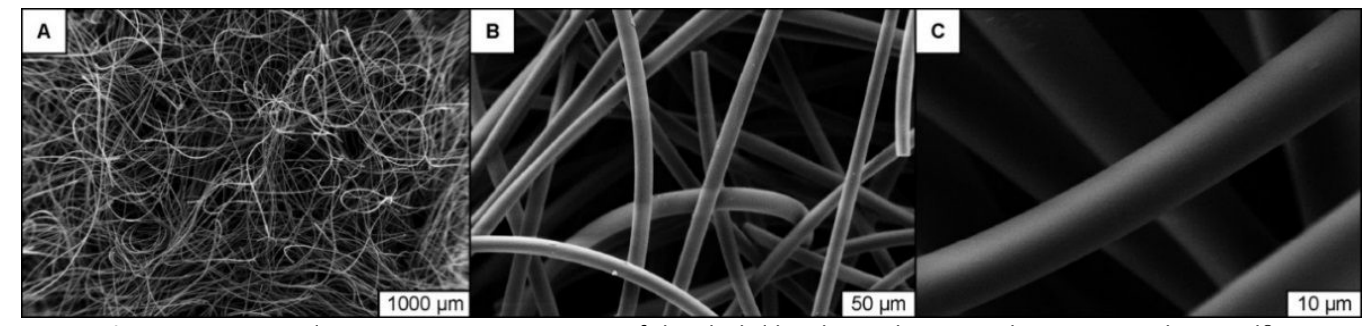

Fig. S2 Scanning electron Microscopy images of the cloth-like electrode material ACN-157-15 by Kynol ${ }^{\circledR}$. 
Figure S3 summarizes the subtraction procedure to obtain only the pore scattering contribution of the electrode material. The measurement was performed in a standard quartz cuvette by Hellma ${ }^{\circledR}$, with a path length of $1 \mathrm{~mm}$. Figure S3a shows the volume normalized scattering curves of the dry carbon electrode $\left(\mathrm{ACN}-157-15\right.$ by $\left.\mathrm{Kyno}^{\circledR}\right)$, deuterated toluene and the wetted carbon electrode. Since the fraction $\phi_{t o l}=0.82$ of toluene in the beam is known, the subtraction of the toluene scattering curve times $\phi_{\text {tol }}$ from the wetted carbon curve yields the residual scattering of the carbon matrix that will be always present. This scattering contribution arises from bulk density fluctuations and inaccessible pores. Figure S3b shows again the unprocessed carbon curve (black circles), the carbon curve where the background curve (Carb Bckgr.) is subtracted and the background curve itself (red circles). The latter curve is used for the processing of the SANS curve in figure $\mathbf{5}$ of the main manuscript. However, the scattering Intensity still exhibits two contribution, namely the pore scattering and the lateral fluctuation scattering with its amplitude $B_{f l}$. Since the latter contribution scales with $q^{-2}$ and the surface scattering of the pores scales with $q^{-4}$ for high $q$ values, a plot of $q^{4} \mathrm{~d} \Sigma_{\mathrm{m}} / \mathrm{d} \Omega(q)$ vs. $q^{2}$ (figure S3c) can be used to extract the mass normalized Porod constant $P_{\mathrm{m}}$ and the parameter $B_{f l}$. For more details see ref ${ }^{15}$. Finally, Figure $\mathbf{S 3 d}$ shows the unprocessed dry carbon curve (black circles), the constant background corrected curve (green circles) and the lateral fluctuation corrected curve (orange circles) that scales with $q^{-4}$ for large q-values. The scattering contribution of the lateral fluctuations is also shown as solid green curve. The parameter $l_{R}$ is set to $1 \mathrm{~nm}$.
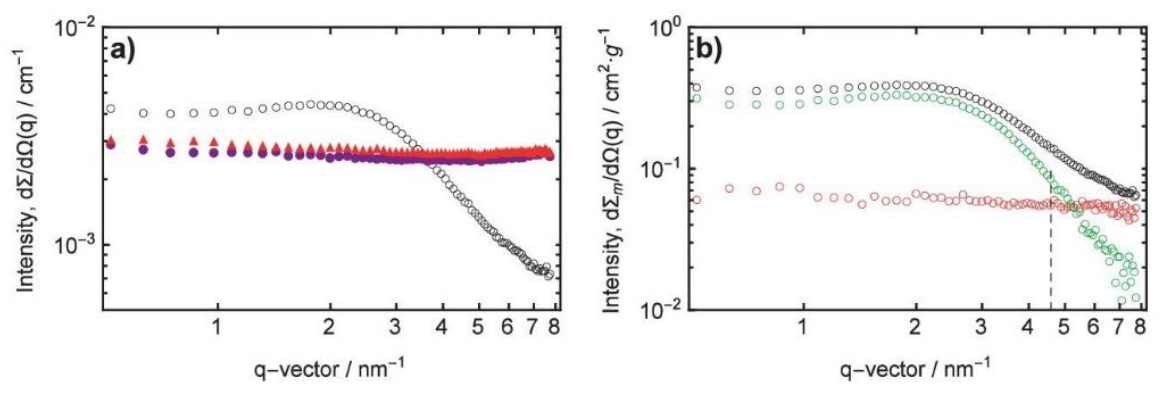

corr. Carb


\begin{tabular}{|c|c|c|c|}
\hline$\phi_{\text {tol }}$ & $\mathrm{m}_{\mathrm{C}, \text { Beam }} / \mathrm{mg}$ & $\rho_{\text {sk }} / \mathrm{g} / \mathrm{cm}^{3}$ & $\rho_{\text {app }} / \mathrm{g} / \mathrm{cm}^{3}$ \\
\hline 0.82 & 2.6 & 2.00 & 0.228 \\
\hline$\rho_{\mathrm{f}} / \mathrm{g} / \mathrm{cm}^{3}$ & $B_{\mathrm{fl}} / \mathrm{cm}^{2} \cdot \mathrm{g}^{-1} \mathrm{~nm}^{-2}$ & $P_{\mathrm{m}} / \mathrm{cm}^{2} \cdot \mathrm{g}^{-1} \cdot \mathrm{nm}^{-4}$ & $\mathrm{~S} / \mathrm{m} / \mathrm{m}^{2} \cdot \mathrm{g}^{-1}$ \\
\hline 0.0103 & 0.51 & 0.0168 & 937 \\
\hline
\end{tabular}

Fig. S3 Summary of the small angle neutron scattering data processing steps of the microporous carbon electrode ACN-157-15 by Kynol. a) Scattering volume normalized SANS curves of the dry carbon electrode (dried at $120^{\circ} \mathrm{C}$ in vacuum oven for $24 \mathrm{~h}$ ), carbon electrode wetted with deuterated toluene and pure deuterated toluene. b) Mass normalized scattering curves of the dry electrode (unprocessed, open black circles), the constant carbon scattering background (red open circles) and the background corrected carbon curve (green open circles). c) $q^{4} \cdot /(q)$ vs. $q^{2}$ plot to obtain the scattering contribution of the lateral fluctuations $B_{\mathrm{fl}}$ in the carbon matrix and the Porod constant $P_{\mathrm{m}}$ from the background corrected curve in b). The black vertical dashed line in b) and c) marks the minimum $q$-value that was used for the linear fit in c). d) Summary of both subtraction steps. Original carbon curve (open black circles), background corrected curve (open green symbols) and lateral fluctuation corrected curve (open orange symbols). 
Figure S4 summarizes the SANS curves of the last three cycles in the charged and discharged state. For comparison, the scattering curve of the dry carbon electrode is also included in the plot. The curves coincide and therefore demonstrate a high degree of reproducibility.

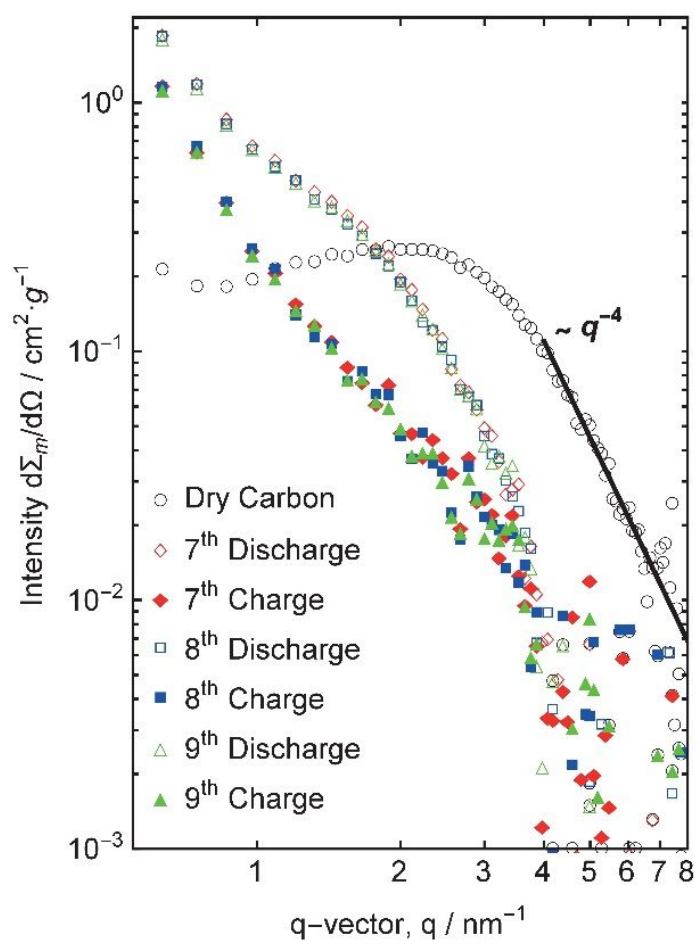

Fig S4 Summary of the SANS curves of the fully charged and discharged states of the last three cycles

Figure S5 summarizes the fit results obtained with equation 3-7.


Fig. S5 Summary of all fit parameters obtained with equations 3-7. From top row to bottom: Cell voltage, average residuum, scattering intensity amplitude, particle diameter, polydispersity and Porod constant. 
Figure S6 summarizes the small angle scattering intensities of dry carbon and sulfur loaded (63wt\%) carbon. The scattering intensity is decreased by a factor of 0.71 which is in excellent agreement with a calculated value of 0.69 . This results shows, first, that sulfur can fully fill the carbon host micropores by melting intrusion and, second, the characteristic scattering signal of the carbon micropores is mapped by a constant factor as illustrated in figure $1 \mathrm{~d}$.

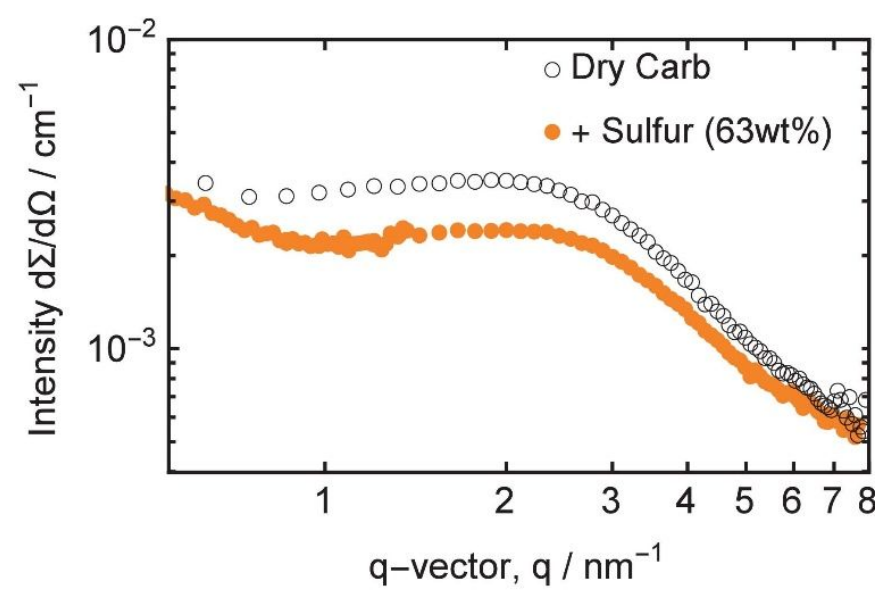

Fig. S6 Comparison of small angle scattering intensity of dry carbon and sulfur loaded (63wt\%) carbon. The change in scattering contrast factor in the micropores causes a vertical shift of the scattering intensity by a factor of 0.71 which is in excellent agreement with the calculated contrast factor change of 0.69 .

The wetting process of the first 10 hours of the operando experiment is illustrated in figure S7.

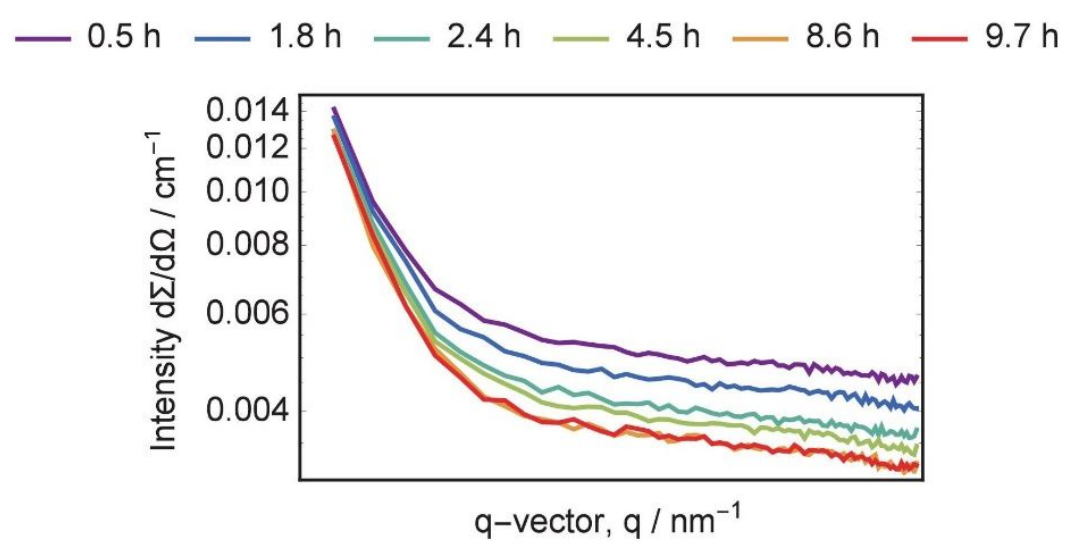

Fig. $\mathbf{S 7}$ Plot of not processed scattering intensities of the first 9.7 hours of the experiment to illustrate the wetting process. The numbers in the legend represent the hours of the experiment. 
Figure $\mathbf{8} 8$ shows the EIS data as a the Nyquist plot of the charged (a) and discharged states (b) of cycles 1-3 and 8-10. The intermediate EIS spectra of the $8^{\text {th }}$ cycle are shown in (c) and (d). The equivalent circuit for the data analysis is shown at the bottom of figure S8 (e).

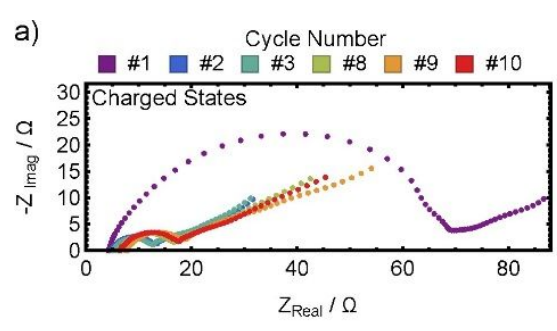

c)
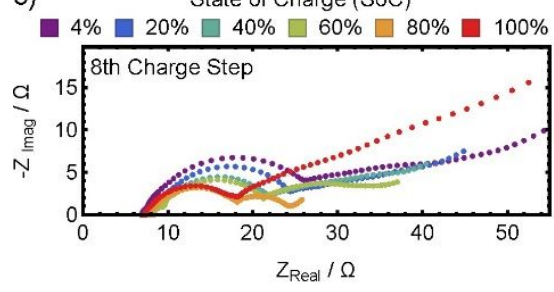

b)



d)

d) State of Discharge (SoD)
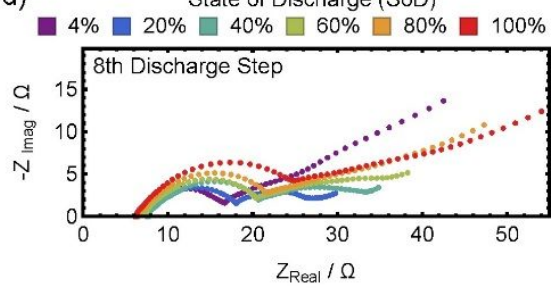

e)

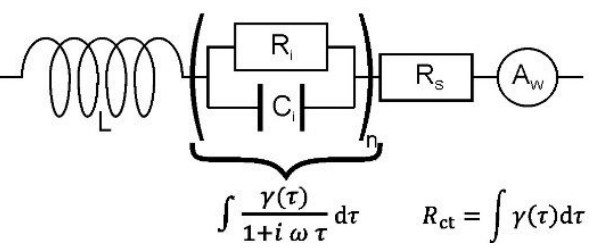

Fig. S8 EIS spectra represented with a Nyquist plot. a) EIS spectra in the charged state for various cycle numbers. b) EIS spectra in the discharged state for various cycle numbers. c) The EIS spectra in the intermediate states of the $8^{\text {th }}$ charge step at different state of charge (SoC). d) The EIS spectra in the intermediate states of the $8^{\text {th }}$ discharge step at different state of discharge (SoD). The bottom row shows the equivalent circuit model that was used for the fitting procedure. It consists of an inductance $(L)$, a solution resistance $\left(R_{S}\right)$, a Warburg impedance $\left(A_{W}\right)$ and a distribution of RC-unit that represent the distribution of relaxation times $\gamma(\tau)$. The values $R_{c t}$ is the charge transfer resistance. 